\title{
Detection of protein concentrations with chronopotentiometry
}

\author{
P. Bergveld*, J. C. T. Eijkel \& W. Olthuis \\ MESA Research Institute, University of Twente, PO Box 217, 7500AE Enschede, The Netherlands
}

(Received 28 February 1996; revised version received 1 April 1997; accepted 1 April 1997)

\begin{abstract}
The chronopotentiogram of a bare $1 \mathrm{~mm}^{2} \mathrm{Ag} / \mathrm{AgCl}$ electrode shows a transition time in the order of $9 \mathrm{~s}$ at a current density of $-25 \mathrm{~A} / \mathrm{m}^{2}$ at $\mathrm{pCl}$ $=2$. At this time the concentration of $\mathrm{Cl}^{-}$ions is completely depleted, a phenomenon that can be explained by using the Nernst-Planck flux equations. Covering the electrode with an $8 \mu \mathrm{m}$ thick membrane of polystyrene beads and repeating the chronopoteniometric experiment results in a decrease of the transition time by a factor of 50 . This can be explained by the Donnan effect resulting from the fixed negative membrane charge originating from the acidic groups of the polystyrene. Modulating the fixed charge by adsorbing protein molecules inside the porous membrane from a sample solution results in a variation of the transition time as a function of the sample protein concentration. As an example, it is shown that positively charged lysozyme neutralizes the initial negative membrane charge, thus increasing the transition time. The advantage of this type of protein concentration measurement is its simplicity: only a membrane-covered electrode is needed through which a current is sent, and from the resulting electrode potential change the transition time is determined. (C)1997 Elsevier Science Limited
\end{abstract}

Keywords: chronopotentiometry, Donnan effect, transition time

\section{INTRODUCTION}

In the field of biosensor research, the development of immunosensors has received specific attention, not only because of the clinical needs to detect antibody or antigen molecules, but also because of the inherent selectivity of an antibody/antigen reaction. The antibody can be considered as a molecular-recognition complex that binds reversibly with a specific antigen, which can be almost any macromolecule that induces an immunoresponse (Turner et al., 1987; Hall, 1990). So any sensor that can be provided

*To whom correspondence should be addressed. with a layer of antibodies can be regarded as the ultimate achievement in the field of biosensor research. Many attempts have been made to follow this route, either in a direct way or in an indirect way, in case the direct way appears to be too difficult. In the latter case label molecules are linked to the antigens, to carry detectable species to the sensor, such as enzymes, fluorescent molecules, etc. More interesting is of course the direct detection of the immunoreaction at the sensor surface. Examples can be found in the area of optical, gravimetric and electrochemical sensors. The latter type of sensors has always been attractive, because of their inherent simplicity. With potentiometric sensors a voltage is measured, with amperometric sensors a current 
is measured and with conductimetric sensors an impedance is measured. In nearly all cases the approach has, up to now, been rather conventional: one tries to measure a thermodynamic and/or kinetic equilibrium state, which should be modulated by an immunoreaction at the surface of the sensor. Therefore, many papers in this field of research have been devoted to the investigation of changes in electrical parameters in a membrane containing antibody molecules before and after incubation in a sample with antigen molecules (Turner et al., 1987; Hall, 1990). As soon as a new type of electrochemical sensor became available, experiments were carried out with this sensor provided with a membrane containing specific antibodies. The immunoFET is an example of this approach (Turner et al., 1987; Hall, 1990; Bergveld, 1991).

Basically, it can not, however, be expected that the measurement of electrical parameters in a static way will be successful, because the conformation of antibody molecules, or protein molecules in general, is such that even the most densely packed layer of these macromolecules is in fact still a layer of electrolyte. The only effect of the presence of the usually charged macromolecules in the membrane is a redistribution of the small cations and anions, known as the Donnan effect. This will result in Donnan potentials with respect to the surrounding electrolyte, but in a symmetrical way. This means that a potentiometric sensor will usually measure the sum of a positive and an equal, but negative, Donnan potential. These compensating effects are inherent to static measurements (Turner et al., 1987; Hall, 1990).

An alternative to the static measurements is the application of dynamic measurements, detecting the response of a system in equilibrium to a change in a system parameter. This concept can be classified as stimulus/response measurements. An unknown and in a direct way unmeasurable thermodynamic equilibrium, for instance a Donnan equilibrium, is disturbed, and the response to a change in one of the system parameters is measured. Using an appropriate model of the system behaviour the static parameter which could not directly be measured can be determined.

It is the aim of this paper to show that the presence of protein molecules, covering an electrode, can be detected by a dynamic experiment, known as chronopotentiometry. Beside the experi- mental results consisting of chronopotentiograms of a bare and a lysozyme covered $\mathrm{Ag} / \mathrm{AgCl}$ electrode, a theoretical description will be presented to explain the results and to elucidate the system.

\section{THE CHRONOPOTENTIOMETRIC RESPONSE OF A Ag/AgCl ELECTRODE}

During a chronopotentiometric measurement, a constant current is sent between the $\mathrm{Ag} / \mathrm{AgCl}$ electrode and a counter electrode placed in the bulk electrolyte. The $\mathrm{Ag} / \mathrm{AgCl}$ electrode is used as the anode and at its surface the following reaction takes place:

$$
\mathrm{Ag}+\mathrm{Cl}^{-} \rightarrow \mathrm{AgCl}+\mathrm{e}^{-}
$$

The chloride ions needed in this reaction will be taken up from the solution. In the case that the mass transport can only partially replenish these ions, a local chloride depletion is created. An electrode which is able to modulate the concentration of chemical species in its surroundings is referred to as a chemical actuator, the opposite of a chemical sensor. $\mathrm{A} \mathrm{Ag} / \mathrm{AgCl}$ electrode can be used both as a sensor and as an actuator. When, during the actuation, the potential of the $\mathrm{Ag} / \mathrm{AgCl}$ electrode is measured in time versus a reference electrode, the gradual chloride depletion will show itself in a potential change of this electrode. A typical $\mathrm{Ag} / \mathrm{AgCl}$ chronopotentiometric response is shown in Fig. 1.

The equilibrium potential at $t<0$ is determined by the electrolyte chloride concentration according to the Nernst equation. The initial ohmic potential rise is determined by the various resistances to current transport resulting from, for

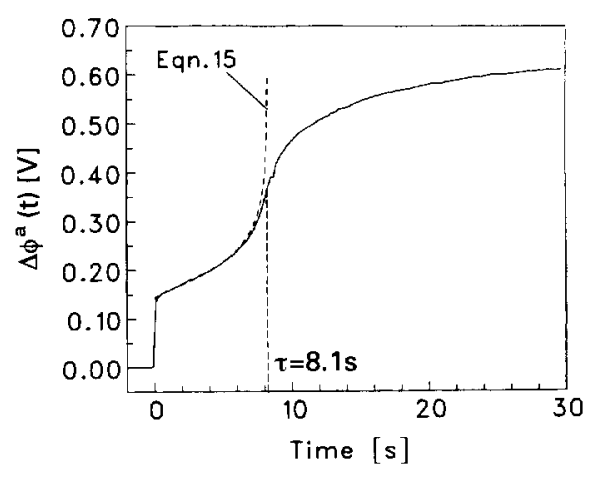

Fig. 1. Theoretical constant current chronopotentiogram of a $\mathrm{Ag} / \mathrm{AgCl}$ anode, showing the various processes that are associated with the measured potential change. 
example, the electrolyte and $\mathrm{AgCl}$ bulk material. The form of the curve following the initial ohmic potential rise can be derived from the $\mathrm{Cl}^{-}$masstransport equations. The behaviour of the system will be considered in the presence of $10 \mathrm{mM} \mathrm{KCl}$ in the electrolyte.

In the absence of supporting electrolyte, both the migration and diffusion contributions to the transport of $\mathrm{K}^{+}$and $\mathrm{Cl}^{-}$have to be considered, and the Nernst-Planck flux equations must be used to calculate their ionic fluxes $J$. The current density $j$ will be composed of the flux contributions $J$ of both ions:

$$
j=\mathrm{F} \cdot\left(J_{\mathrm{K}}(t, x)-J_{\mathrm{Cl}}(t, x)\right)
$$

where $j\left(\mathrm{~A} / \mathrm{m}^{2}\right)$ is the current density, negative in the case of chloride uptake, $\mathrm{F}(\mathrm{C} / \mathrm{mol})$ is the Faraday constant and $J_{i}\left(\mathrm{~mol} \mathrm{~m}^{-2} \mathrm{~s}^{-1}\right)$ is the flux of species $i$.

Assuming that only $\mathrm{K}^{+}$and $\mathrm{Cl}^{-}$contribute to the space charge in the electrolyte, the bulk electroneutrality condition implies equal concentrations of both ions. From Eq. (1), the bulk electroneutrality condition and the Nernst-Planck equations, the fluxes of both ions can be derived as

$$
\begin{aligned}
& J_{\mathrm{Cl}}(t, x)=-t_{\mathrm{Cl}}^{\mathrm{e}} \cdot \frac{j}{\mathrm{~F}}-D^{\mathrm{e}} \cdot \frac{\partial c_{\mathrm{KCl}}(t, x)}{\partial x} \\
& J_{\mathrm{K}}(t, x)=t_{\mathrm{K}}^{\mathrm{e}} \cdot \frac{j}{\mathrm{~F}}-D^{\mathrm{e}} \cdot \frac{\partial c_{\mathrm{KCl}}(t, x)}{\partial x}
\end{aligned}
$$

where $t_{\mathrm{Cl}}^{c}$ is the transport number of $\mathrm{Cl}^{-}$in the electrolyte, $t_{\mathrm{K}}^{\mathrm{e}}$ is the transport number of $\mathrm{K}^{+}$in the electrolyte, $c_{\mathrm{KCl}}\left(\mathrm{mol} / \mathrm{m}^{3}\right)$ is the $\mathrm{KCl}$ concentration and $D^{\mathrm{e}}$ is the mean diffusion coefficient of $\mathrm{KCl}$ in the electrolyte $\left(2.0 \times 10^{-9} \mathrm{~m}^{2} / \mathrm{s}\right)$, defined as

$$
D^{\mathrm{e}}=\frac{2 D_{\mathrm{K}}^{\mathrm{e}} D_{\mathrm{Cl}}^{\mathrm{e}}}{D_{\mathrm{K}}^{\mathrm{e}}+D_{\mathrm{C} 1}^{\mathrm{e}}}
$$

The transport number $t_{i}$ of an ionic species $i$ is the fraction of the electrical current carried by this species and equals

$$
t_{i}=\frac{c_{i} D_{i}}{\Sigma c_{i} D_{i}}
$$

Owing to the equal mobilities of $\mathrm{K}^{+}$and $\mathrm{Cl}$ - , the transport number of chloride in the electrolyte is 0.5 . In the actuator it is 1 , when a current efficiency of 1 is assumed for the $\mathrm{Cl}^{-}$actuation. This implies that the charging current for the double layer of the actuator is assumed to be negligible, which can be proven to be valid.

Inspecting Eqs (2) and (3), the first terms on the right-hand side represent the migration fluxes and the second the diffusion fluxes. The migration flux is constant within every phase, because it only depends on the transport number and the current density. Since the $\mathrm{Cl}^{-}$transport number is lower in the electrolyte than in the electrode, in the electrolyte an additional diffusion flux of $\mathrm{Cl}^{-}$is required, which creates a continuously growing $\mathrm{KCl}$ depletion layer. This process, together with the potential change in which it results, is known as concentration polarization. It always occurs when transport numbers differ between phases. An equation describing the $\mathrm{KCl}$ concentration profile in the electrolyte can be derived by applying the continuity equation to Eq. (2) or Eq. (3). The continuity equation is given by

$$
\frac{\partial c_{i}(t, x)}{\partial t}=-\frac{\partial J_{i}(t, x)}{\partial x}
$$

Since the spatial derivative of the current density is zero, the migration terms drop and the following diffusion equation is obtained (Bard \& Faulkner, 1980; Audinos \& Pichelin, 1988; Levich, 1969),

$$
\frac{\partial c_{\mathrm{KCl}}(t, x)}{\partial t}=D^{\mathrm{e}}\left(\frac{\partial^{2} c_{\mathrm{KCl}}(t, x)}{\partial x^{2}}\right)
$$

The following initial and boundary conditions apply (Bard \& Faulkner, 1980; Levich, 1969),

$$
\begin{aligned}
& t=0, \text { all } x, c_{\mathrm{KCl}}(t, x)=c^{\mathrm{e}} \\
& x=\infty, \text { all } t, c_{\mathrm{KCl}}(t, x)=c^{\mathrm{e}} \\
& x=0, t>0, \frac{\partial c_{\mathrm{KCl}}(t, 0)}{\partial x}=\frac{j \cdot\left(t_{\mathrm{C} 1}^{\mathrm{a}}-t_{\mathrm{Cl}}^{\mathrm{e}}\right)}{\mathrm{F} D^{\mathrm{e}}}
\end{aligned}
$$

where $t_{\mathrm{Cl}}^{\mathrm{a}}$ is the transport number of $\mathrm{Cl}^{-}$in the actuator, which equals unity. Boundary condition (10) for the actuator-electrolyte interface results from equating the local $\mathrm{Cl}^{-}$fluxes in the actuator and the electrolyte (given by Eq. (2))

$$
\frac{-t_{\mathrm{C}}^{\mathrm{a}} j}{\mathrm{~F}}=\frac{-t_{\mathrm{C}}^{\mathrm{e}} j}{\mathrm{~F}}-D^{\mathrm{e}} \frac{\partial c_{\mathrm{KCl}}(t, 0)}{\partial x}
$$

Solution of Eq. (7) under the conditions of Eqs (8)-(10) gives for the $\mathrm{KCl}$ profile in the electrolyte (Bard \& Faulkner, 1980):

$$
c_{\mathrm{KCl}}(t, x)=c^{\mathrm{e}}+\frac{j\left(1-t_{\mathrm{C}}^{\mathrm{e}}\right)}{\mathrm{FD}^{\mathrm{e}}}\left\{2\left(\frac{\mathrm{D}^{\mathrm{e}} \mathrm{t}}{\pi}\right)^{1 / 2}\right.
$$




$$
\left.\exp \left(-\frac{x^{2}}{4 D^{\mathrm{e}} t}\right)-x \cdot \operatorname{erf} c\left[\frac{x}{2\left(D^{\mathrm{e}} t\right)^{1 / 2}}\right]\right\}
$$

The time in which the $\mathrm{KCl}$ concentration at the actuator surface reaches zero is characteristic for the system. It is called the transition time $\tau$ [s], and follows from Eq. (12) as the Sand equation

$$
\tau=\left[\frac{\mathrm{F} c_{\mathrm{Cl}}^{\mathrm{e}}}{2 j\left(1-t_{\mathrm{Cl}}^{\mathrm{e}}\right)}\right]^{2} \cdot D^{\mathrm{e}} \pi=t_{\text {Sand }}
$$

With use of the transition time $\tau$, the $\mathrm{KCl}$ concentration at the actuator surface can be conveniently expressed as

$$
c_{\mathrm{KCl}}(t, 0)=c^{\mathrm{e}} \cdot\left(1-\sqrt{\frac{t}{\tau}}\right)
$$

This equation can be used for the derivation of the potential of the $\mathrm{Ag} / \mathrm{AgCl}$ electrode in time, the chronopotentiogram. When rapid electron transfer is assumed at the actuator surface, its response to the local $\mathrm{Cl}^{-}$concentration can be derived from Eq. (14) and the Nernst relation (Bard \& Faulkner, 1980). The contribution of a diffusion potential is neglected, since $\mathrm{KCl}$ is the diffusing salt. The final potential response of the $\mathrm{Ag} / \mathrm{AgCl}$ electrode created over the bulk electrolyte, the diffusion layer, the interface and the $\mathrm{AgCl}$ bulk material is (Eijkel, 1995):

$$
\begin{aligned}
& \Delta \Phi^{\mathrm{a}}(t)=\frac{-j}{\lambda c^{\mathrm{e}}}\left(d-\sqrt{\pi D^{\mathrm{e}} t}\right)+\left(\pi t_{\mathrm{Cl}}^{\mathrm{e}}+1\right) \\
& \frac{\mathrm{R} T}{\mathrm{~F}} \ln \left(\frac{1}{1-\sqrt{(t / \tau)}}\right)+\Phi^{\Omega, \mathrm{a}}(t)
\end{aligned}
$$

where $\lambda$ is the equivalent conductance of $\mathrm{KCl}$ $\left[\Omega^{-1} \mathrm{~m}^{2} \mathrm{~mol}^{-1}\right]$, and $d[\mathrm{~m}]$ is the distance between actuator surface and reference electrode.

In Fig. 2 the actuator potential change, $\Delta \Phi^{\mathrm{a}}(t)$, calculated with Eq. (15) is shown.

The potential rises sharply when $t$ approaches the transition time $\tau$. At that moment the almost total depletion of $\mathrm{KCl}$ at the actuator surface (see Eq. (14)) causes a rapid increase of both the ohmic term and the sensor chloride response. A zero chloride concentration would correspond to an infinitely positive potential and can not be reached in real situations. Instead, as was shown

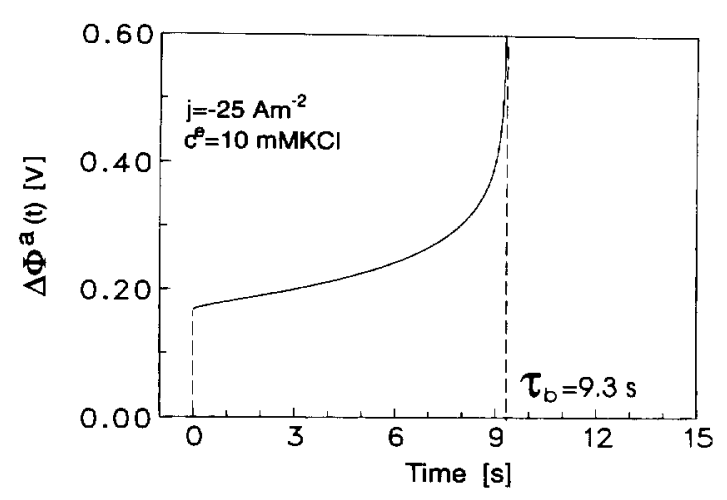

Fig. 2. The analytically derived (Eq. (15), $d=I \mathrm{~mm}$ ) chronopotentiogram at a current density of $-25 \mathrm{~A} / \mathrm{m}^{2}$ for a $10 \mathrm{mM} \mathrm{KCl}$ solution. Past the transition time $\tau$ a new potential plateau is expected owing to the occurrence of other processes $\left(\mathrm{O}_{2}\right.$ or $\mathrm{Ag}_{2} \mathrm{O}$ formation; see Figure 1); $D^{\mathrm{e}}=2.0 \times 10^{-9} \mathrm{~m}^{2} / \mathrm{s}$.

in Fig. 1, at a sufficiently positive potential either the formation of $\mathrm{Ag}_{2} \mathrm{O}$ or oxygen evolution will occur. Then the current efficiency for the actuation of $\mathrm{Cl}^{-}$becomes less than one so that boundary condition Eq. (10) changes, and the electrode potential is no longer described by Eq. (15). Such a different oxidation process generally will cause a new potential plateau to occur.

\section{THE CHRONOPOTENTIOMETRIC RESPONSE OF A MEMBRANE-COVERED $\mathrm{Ag} / \mathrm{AgCl}$ ELECTRODE}

The $\mathrm{Ag} / \mathrm{AgCl}$ electrode as described in the previous section is now considered to be covered with a membrane consisting of polystyrene beads in an agarose gel. This membrane may contain adsorbed protein molecules which exhibit fixed positive or negative charges depending on the electrolyte $\mathrm{pH}$. A Donnan equilibrium will establish itself between this membrane and the adjacent solution, characterized by a distribution of the diffusible ions according to the Donnan ratio, $r_{\mathrm{D}}$ (Donnan, 1924).

The Donnan ratio depends on the fixed charge concentration, $z c_{\text {fixed }}^{\mathrm{m}}$, and the salt concentration, $c^{e}$ :

$$
r_{\mathrm{D}}=\left(\frac{c_{\mathrm{K}}^{\mathrm{e}}}{c_{\mathrm{K}}^{\mathrm{m}}}\right)=\left(\frac{c_{\mathrm{Cl}}^{\mathrm{m}}}{c_{\mathrm{Cl}}^{\mathrm{e}}}\right)=\frac{\sqrt{4\left(c^{\mathrm{e}}\right)^{2}+\left(z c_{\mathrm{fixed}}^{\mathrm{m}}\right)^{2}}+z c_{\mathrm{fixed}}^{\mathrm{m}}}{2 c^{e}}
$$

Consequently, between membrane and electrolyte a Donnan potential $\Phi_{D}$ exists 


$$
\Phi_{\mathrm{D}}=\Phi^{\mathrm{m}}-\Phi^{\mathrm{e}}=\frac{\mathrm{R} T}{\mathrm{~F}} \ln r_{\mathrm{D}}
$$

The Donnan equilibrium results in a high concentration of counterions and a low concentration of co-ions inside the membrane. As is shown in Fig. 3, this results on current actuation in the development of concentration profiles that differ for a positively and a negatively charged membrane.

Owing to the high $\mathrm{Cl}^{-}$concentration in a positively charged membrane, the chronopotentiogram of such a system will not deviate from that of a bare $\mathrm{Ag} / \mathrm{AgCl}$ electrode: the diffusion layer and the subsequent depletion will occur in the electrolyte. However, the low $\mathrm{Cl}^{-}$concentration in a negatively charged membrane will quickly be depleted during current actuation, resulting in a drastic reduction of the transition time:

$$
\tau^{\mathrm{m}}=\left[\frac{\mathrm{F} c_{\mathrm{Cl}}^{\mathrm{m}}}{2 j\left(1-t_{\mathrm{Cl}}^{\mathrm{m}}\right)}\right]^{2} \cdot D^{\mathrm{m}} \pi
$$

This transition time for chloride depletion at the interface of membrane and actuator can be deduced by realizing that the chloride transport number in the membrane is determined by the Donnan distribution between electrolyte and membrane:

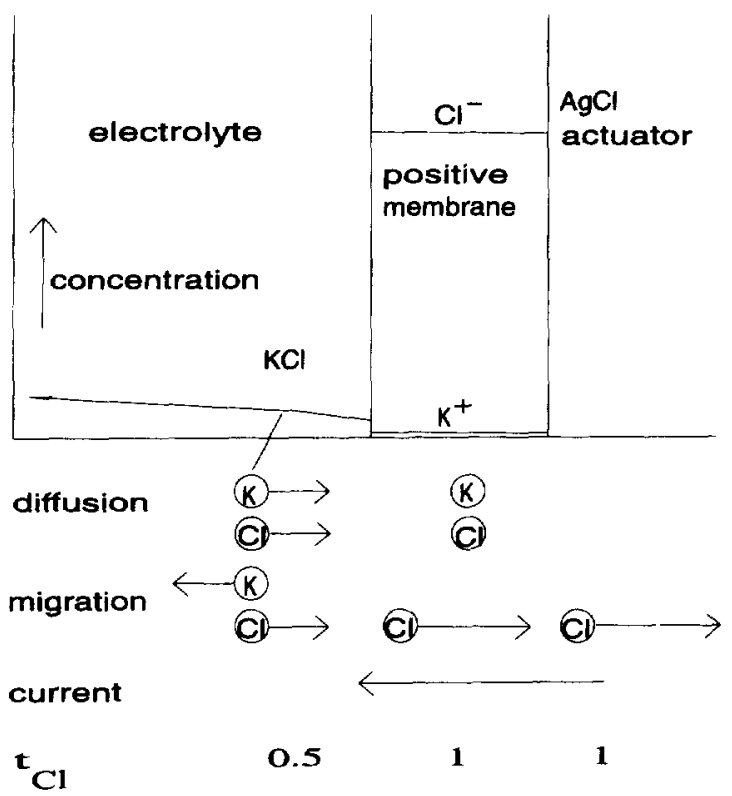

$$
t_{\mathrm{Cl}}^{\mathrm{m}}=\frac{r_{\mathrm{D}}^{2}}{r_{\mathrm{D}}^{2}+1}
$$

so that, with the use of the Donnan ratio to calculate the membrane chloride concentration, the transition time at the membrane/actuator interface, $\tau^{\mathrm{m}}$, becomes

$$
\tau^{\mathrm{m}}=\left[\frac{\mathrm{F} c_{\mathrm{Cl}}^{\mathrm{e}}}{2 j} \cdot\left(r_{\mathrm{D}}^{2}+1\right)\right]^{2} \cdot r_{\mathrm{D}}^{2} \cdot D^{\mathrm{m}} \pi
$$

Equation (20) for $\tau^{\mathrm{m}}$ differs in three aspects from Eq. (13) for the transition time $\tau$ in the absence of a membrane, namely in the Donnan ratio term between the square brackets that stems from the difference in the chloride transport numbers between electrolyte and membrane, in the Donnan ratio term outside the brackets of Eq. (20) that stems from the membrane chloride concentration, and in the diffusion coefficient. Bearing in mind that the Donnan ratio depends on the fixed-charge concentration, the dependence of the transition time $\tau^{\mathrm{m}}$ on the fixed charge can be evaluated. In a strongly negatively charged membrane where $r_{\mathrm{D}} \ll 1, \tau^{\mathrm{m}}$ will be much smaller than $\tau$. Depletion will occur more rapidly at the interface of membrane and actuator (see Fig. 3). This large difference between $\tau^{\mathrm{m}}$ and $\tau$ is mainly caused by the low chloride concentration in the membrane represented by the $r_{\mathrm{D}}^{2}$ factor in Eq.

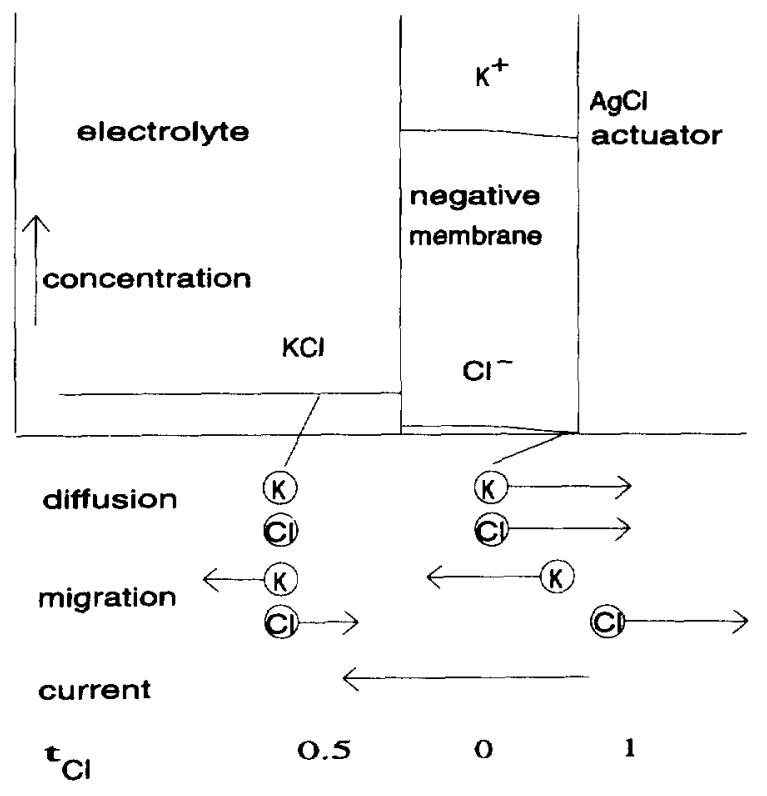

Fig. 3. $\mathrm{KCl}$ concentration profiles during chronopotentiometry with the measuring device. Differences in the chloride transport number cause $\mathrm{KCl}$ depletion at the interface of electrolyte and membrane when the membrane is positive, and at the interface of membrane and actuator when the membrane is negative. 
(20), and less by the difference in $\mathrm{Cl}^{-}$transport numbers represented by the term between the square brackets. With increasing negative fixed charge in the membrane, both the membrane chloride concentration and $\tau^{\mathrm{m}}$ will approach zero. Such systems will behave as a chloride current rectifier, just as a combination of oppositely charged membranes has rectifying properties (de Körösy, 1975), since current can only pass at a cathodic polarization of the $\mathrm{Ag} / \mathrm{AgCl}$ electrode. The membrane diffusion coefficient, $D^{\mathrm{m}}$, has been determined as $0.8 \times 10^{-9} \mathrm{~m}^{2} / \mathrm{s}$ for the membranes used (Eijkel et al., 1997).

The chronopotentiometric signal from which the transition time can be obtained is the potential change of the actuator electrode versus a reference electrode in the bulk electrolyte. The actuator potential change will consist, as in the case of a bare electrode, of ohmic potential rises, diffusion potentials and the response of the $\mathrm{Ag} / \mathrm{AgCl}$ electrode to the $\mathrm{Cl}^{-}$concentration at its surface, with in addition for this case the change of the Donnan potential at the electrolyte/membrane interface:

$$
\Delta \Phi^{\mathrm{a}}(t)=\Sigma \Phi^{\Omega}(t)+\Sigma \Phi^{\mathrm{dif}}(t)+\Delta \Phi_{\mathrm{D}}(t)+\Delta \Phi^{\mathrm{ion}}(t)
$$

With strongly negatively charged membranes, $\mathrm{KCl}$ depletion will occur only at the membrane/actuator interface as is illustrated in Fig. 3. In this case the salt concentration at the electrolyte/membrane interface does not change, and the Donnan term and the term for the ohmic rise in the electrolyte in Eq. (21) do not change in time. Since the ohmic rise in the membrane will be negligible owing to the large counterion concentration which is not depleted (see Fig. 3), and since it can be assumed that all diffusion potentials are zero since $\mathrm{KCl}$ is used, Eq. (21) reduces to

$$
\Delta \Phi^{\mathrm{a}}(t)=\Phi^{\Omega, \mathrm{e}}+\Phi^{\Omega, \mathrm{a}}(t)-\frac{\mathrm{R} T}{\mathrm{~F}} \cdot \ln \left(1-\sqrt{\frac{t}{\tau^{\mathrm{m}}}}\right)
$$

Thus, for a strongly negatively charged membrane only two time-dependent terms are left, representing the ohmic potential rise in the actuator and the Nernstian $\mathrm{Cl}^{-}$response of the actuator. The former term is almost constant, as shown above, so that the actuator potential will mainly change because of its Nernstian response to the rapidly decreasing $\mathrm{Cl}^{-}$concentration in the membrane close to the actuator. The result of Eq. (22) is shown in Fig. 4, and comparison with the chronopotentiometric curve in the absence of a membrane as calculated by Eq. (13) and as shown in Fig. 2 shows the large reduction in the transition time (from $9.3 \mathrm{~s}$ to $40 \mathrm{~ms}$ ), corresponding with the change from $\tau$ to $\tau^{\mathrm{m}}$. In addition, the approach of the curve of Fig. 4 towards the transition time is more flat than the one of Fig. 2 owing to the absence of the ohmic rise.

\section{DETECTION OF THE ADSORPTION OF CHARGED MOLECULES ON A NEGATIVELY CHARGED MEMBRANE}

It will be clear that a change in the negative fixed charge concentration of the membrane causes a detectable change of the transition time. Such a change can result from the adsorption of charged molecules, either with positive or negative charge, to the negatively charged membrane. The fixed charge concentration of a polystyrene beads/agarose membrane typically is about $40 \mathrm{mM}$ (Eijkel, 1997), and will change on adsorption of the positively charged protein lysozyme. Thus, adsorption experiments with lysozyme are suitable for verifying the theory given in the previous section.

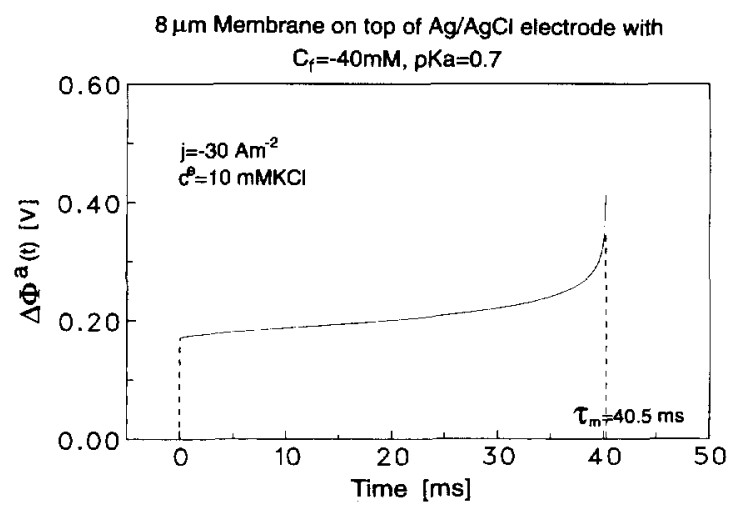

Fig. 4. Analytically derived chronopotentiogram $(j=-$ $30 \mathrm{~A} / \mathrm{m}^{2}$ through a membrane with - $40 \mathrm{mM}$ fixed charge $\left(p K_{\mathrm{a}}=0.7\right)$ in an unbuffered $10 \mathrm{mM} \mathrm{KCl}$ solution of $\mathrm{pH} 7$ ). Past the transition time $\tau^{\mathrm{m}}$, the occurrence of other electrode processes will lead to a second potential plateau. 
Fig. 5 shows the transition time, $\tau^{\mathrm{m}}$, as a function of the concentration of adsorbed lysozyme as calculated from Eqs (16) and (20). The value for the maximal concentration of adsorbed lysozyme was determined by the ion-step measuring method as $5.7 \mathrm{mM}$ (Eijkel, 1995). Considering that at $\mathrm{pH}^{\mathrm{e}} 4$ each lysozyme molecule introduces about 14 positive charges (Tanford \& Wagner, 1954), the maximal fixed charge concentration at this $\mathrm{pH}$ after lysozyme adsorption is $+40 \mathrm{mM}$ after subtraction of the initial $-40 \mathrm{mM}$ of fixed charge. The figure shows the increase of the transition time with the concentration of adsorbed lysozyme until $\tau$ is reached, which is the transition time in the absence of a membrane. It also shows that the change in the transition time is largest at the low current density $j$, which follows directly from Eq. (20).

\section{EXPERIMENTAL}

The chronopotentiometric experiments were performed using devices with a $\mathrm{Ag}$ electrode deposited on a silicon chip. A polyimide window left an area of $1 \mathrm{~mm}^{2}$ uncovered. These chips were mounted on a piece of printed circuit board and encapsulated with Hy-Sol epoxy. A circular area with a diameter of $1.7 \mathrm{~mm}$ was left uncovered. The uncovered silver was partly galvanostatically chloridized by a current of $1 \mu \mathrm{A}$ for $30 \mathrm{~s}$ in a solution of $1 \mathrm{M} \mathrm{KCl}$ at $\mathrm{pH} \mathrm{4}$, to

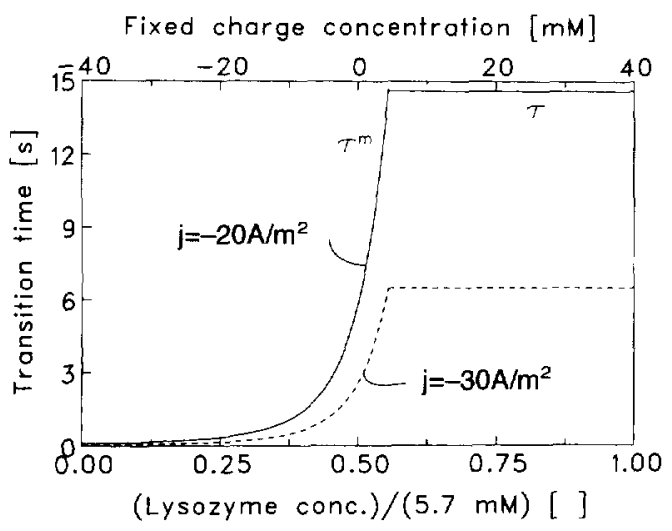

Fig. 5. Calculation using the Eqs (16) and (20) for $\tau^{\mathrm{m}}$ and $E q$. (13) for $\tau$, of the effect of the concentration of adsorbed lysozyme on the observed transition time at two current densities. $\mathrm{pH}^{\mathrm{e}}=4$. The lysozyme concentration at saturated adsorption is $5.7 \mathrm{mM}$. The upper $x$-axis shows the fixed charge concentration after adsorption. create a $\mathrm{Cl}^{-}$response. In the circular area the polystyrene beads/agarose membrane was cast (Schasfoort et al., 1990). The casting solution was prepared by $1: 1$ mixing of a $2.5 \%$ solution of polystyrene beads of $112 \mathrm{~nm}$ diameter (Polysciences) and a $0.25 \%$ solution of agarose (Biorad; $0 \mathrm{Mr}$ ) in demineralized water. After careful mixing at $50^{\circ} \mathrm{C}, 3 \mu \mathrm{l}$ of this solution was deposited in the circular area. Subsequently, the device was cooled at $4^{\circ} \mathrm{C}$ for $12 \mathrm{~h}$ to allow slow evaporation of the water. Membranes cast this way have a thickness of $8 \mu \mathrm{m}$ when measured in dry conditions with a surface profiler (Sloan Dektak). Lysozyme (from hen egg white, Sigma, St Louis, MO) was adsorbed from solutions containing $5,0.5$ and $0.05 \mathrm{mg} / \mathrm{ml}$ in $100 \mathrm{mM} \mathrm{KCl}$ that were buffered at $\mathrm{pH} 7.6$ using $50 \mathrm{mM}$ HEPES. Chronopotentiometric experiments were performed in $10 \mathrm{mM} \mathrm{KCl}$ solutions buffered, respectively, at $\mathrm{pH} 4.0$ or $\mathrm{pH} 4.8$ with acetic acid $(0.5 \mathrm{mM}), \mathrm{pH} 6.2$ with MES $(0.5 \mathrm{mM})$, pH 7.6 with HEPES $(0.5 \mathrm{mM})$ and $\mathrm{pH} 8.1$ with TRIS $(0.5 \mathrm{mM})$. The $\mathrm{pH}$ in each case was adjusted by the addition of $\mathrm{KOH}$. A current was sent between the $\mathrm{Ag} / \mathrm{AgCl}$ electrode and a $\mathrm{Pt}$ counter electrode using a battery-fed current source. During the experiment the potential of the $\mathrm{Ag} / \mathrm{AgCl}$ electrode versus the reference electrode (a saturated calomel electrode, SCE) was measured using a unity gain amplifier with an input impedance of $1 \mathrm{~T} \Omega$. The signal was recorded on a Nicolet 310 Memory Scope.

The transition time $\tau^{\mathrm{m}}$ was estimated in the experiments with a bare membrane by taking the moment of steepest potential rise after the initial ohmic rise. In the experiments where lysozyme adsorption took place, this moment was sometimes difficult to establish. In these experiments the transition time was taken as the moment when the potential exceeded the value of the initial ohmic rise with an added $200 \mathrm{mV}$.

\section{MEASUREMENT RESULTS}

\section{Chronopotentiograms in the absence and presence of a negatively charged membrane}

Fig. 6 shows the chronopotentiogram in the absence of a membrane. The experiment was performed in a solution of $10 \mathrm{mM} \mathrm{KCl}$ and $\mathrm{pH} 4$ with a current density of $-30 \mu \mathrm{A} / \mathrm{mm}^{2}$. The transient $\Delta \Phi^{\mathrm{a}}(t)$ for the $\mathrm{Ag} / \mathrm{AgCl}$ electrode is 


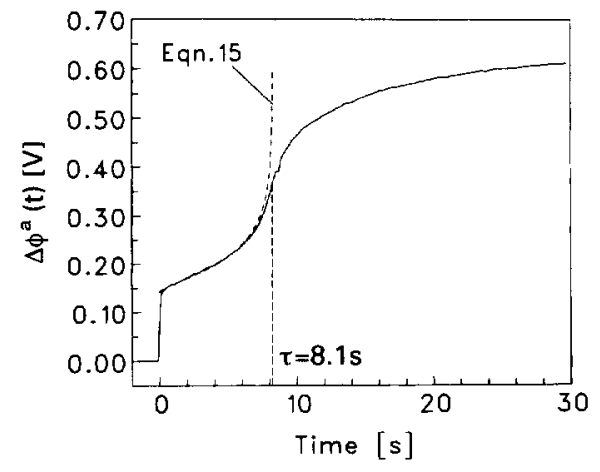

Fig. 6. The $\mathrm{Ag} / \mathrm{AgCl}$ actuator response in the absence of a membrane. A current density of $-30 \mathrm{~A} / \mathrm{m}^{2}$ was sent in a solution of $10 \mathrm{mM} \mathrm{KCl}$ brought at $\mathrm{pH} 4$ with $0.5 \mathrm{mM}$ acetic acid. A fit is shown with the theoretical Eq. (15) $(\tau=8.25 \mathrm{~s})$.

defined relative to its initial equilibrium potential, which is $0.102 \mathrm{~V}$ versus SCE. At $t>0$ the $\mathrm{Ag} / \mathrm{AgCl}$ potential first shows a rapid ohmic rise of $0.13 \mathrm{~V}$, followed by a slower increase associated with the chloride depletion in the neighbouring solution. A new plateau is reached above $0.6 \mathrm{~V}$ versus SCE $(0.7 \mathrm{~V}$ versus NHE), caused by oxygen evolution and/or the formation of silver oxide. The transition time, estimated by the time of steepest potential rise, is $8.1 \mathrm{~s}$. A fit with the theoretical Eq. (15) gives a value of $\tau=$ $8.25 \mathrm{~s}$ and is shown in Fig. 6 as a dashed curve. Clearly, the $\mathrm{Ag} / \mathrm{AgCl}$ potential rise in its first phase is excellently predicted by Eq. (15).

A typical chronopotentiogram of a device with a negatively charged $8 \mu \mathrm{m}$ membrane of polystyrene beads/agarose deposited on the $\mathrm{Ag} / \mathrm{AgCl}$ electrode is shown in Fig. 7.

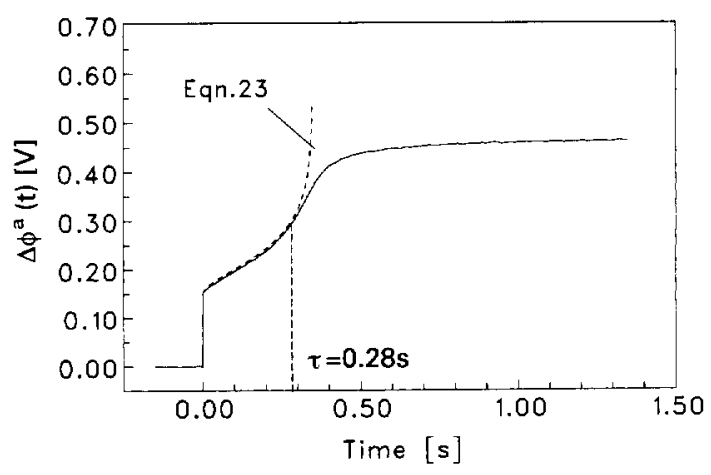

Fig. 7. The response of a $\mathrm{Ag} / \mathrm{AgCl}$ actuator covered by a bare $8 \mu \mathrm{m}$ membrane. Current density $-30 \mathrm{~A} / \mathrm{m}^{2}$, electrolyte $10 \mathrm{mM} \mathrm{KCl}$ brought at $\mathrm{pH} 4$ with $0.5 \mathrm{mM}$ acetic acid. The fit with the theoretical Eq. 23 gives $\tau$

$$
=0.35 \mathrm{~s} \text {. }
$$

The equilibrium potential before the start of the measurement is equal to the value without a membrane, namely $0.102 \mathrm{~V}$ versus SCE. The initial ohmic rise is $0.12 \mathrm{~V}$, which also is about equal to the value in the absence of a membrane. Neither the presence of a membrane nor the thickness of the $\mathrm{AgCl}$ layer are of significant influence on the initial ohmic rise, which implies that it is situated exclusively in the electrolyte. Apparently the $\mathrm{AgCl}$ layers grown have a lower specific resistance than reported in the literature. (At a current density of $-30 \mathrm{~A} / \mathrm{m}^{2}$ the reported resistance of about $5 \times 10^{3} \Omega \cdot \mathrm{m}$ (Ives \& Janz, 1961; Lal et al., 1951) would cause a potential rise of $450 \mathrm{mV}$ at the maximal layer thickness of $3 \mu \mathrm{m}$.)

Also, the position of the reference electrode relative to the actuator does not influence the ohmic rise. This is probably because the area through which the current is transported increases with increasing distance from the actuator, so that mainly the electrolyte area close to the actuator surface contributes to the measured resistance.

The observed transition time of devices with a membrane is $290 \mathrm{~ms}$, which differs much from the device without a membrane $(8 \mathrm{~s})$. In the theoretical section it was shown that this is caused by the rapid chloride depletion at the membrane/actuator interface owing to the low chloride concentration in the membrane. Because the potential rise is too steep, the chronopotentiogram can not be fitted with the theoretical Eq. (22), only by the following Eq. (23), which retains the pre-logarithmic term for the ohmic rise owing to $\mathrm{KCl}$ depletion as deduced for Eq. (15) (Eijkel, 1995):

$$
\Delta \Phi^{\mathrm{a}}(t)=\Phi^{\Omega \cdot \mathrm{e}}+\left(\pi t_{\mathrm{Cl}}^{\mathrm{e}}+1\right) \frac{\mathrm{R} T}{\mathrm{~F}} \cdot \ln \left(\frac{1}{1-\sqrt{\frac{t}{\tau^{\mathrm{m}}}}}\right)
$$

Since the membrane fixed charge stems from polystyrene sulphate groups with $\mathrm{pK}_{\mathrm{a}}=0.7$, it is expected that the fixed-charge concentration will not depend on the $\mathrm{pH}$. To check this expectation, chronopotentiograms of five devices were measured as a function of the $\mathrm{pH}$ at a current density of $-25 \mathrm{~A} / \mathrm{m}^{2}$. Fig. 9 ('bare membrane') shows that the transition time is independent of the $\mathrm{pH}$ $(290 \pm 90 \mathrm{~ms})$, which confirms this expectation. The standard deviation mentioned is the deviation 
of the measurements with the five devices, $90 \mathrm{~ms}$. The standard deviation for subsequent measurements with one device is about $10 \mathrm{~ms}$, and mainly determined by the noise on the potential signal (typically $4 \mathrm{mV}$ top/top). The membranes show a gradual ageing process. When stored in a solution of $10 \mathrm{mM} \mathrm{KCl}$ at $\mathrm{pH} 4.8(0.5 \mathrm{mM}$ acetic acid $)$ for 5 days the transition time gradually increases, approximately $100 \mathrm{~ms} /$ day. This phenomenon can be explained by membrane swelling, causing an increasing membrane diffusion coefficient and a decreasing fixed-charge concentration.

\section{The influence of lysozyme adsorption on the transition time}

Fig. 8 shows a typical chronopotentiogram of a device with an $8 \mu \mathrm{m}$ membrane after incubation for $1 \mathrm{~h}$ in a solution containing $3 \times 10^{-4} \mathrm{M}$ $(5 \mathrm{mg} / \mathrm{ml})$ lysozyme.

Since it is more difficult to determine the moment of steepest potential rise before than after the adsorption of lysozyme, the transition time is determined by taking the moment where the potential passes the initial ohmic rise $+200 \mathrm{mV}$. The transition time proves to be increased from the $290 \pm 90 \mathrm{~ms}$ before the lysozyme adsorption to $9 \pm 1 \mathrm{~s}$ (mean and standard deviation for three devices). The transition time of control devices placed in the same incubation solution but without lysozyme does not increase in the same period. The measured value is close to the theoretical value of $9.4 \mathrm{~s}$ (Eq. (18)) and the measured value of $12 \mathrm{~s}$ for a device without a membrane at this current density. Comparison with Fig. 5 indicates

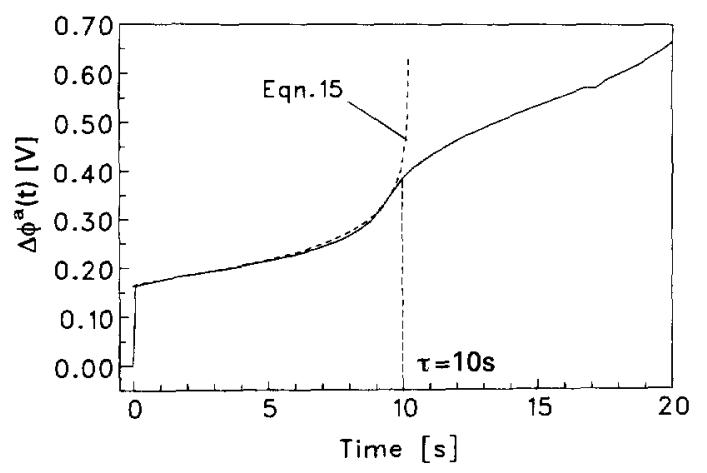

Fig. 8. Chronopotentiogram of a device with membrane incubated for $1 \mathrm{~h}$ in a solution containing $3 \times 10^{-4} \mathrm{M}$ lysozyme. Current density - $25 \mathrm{~A} / \mathrm{m}^{2}$, electrolyte: $10 \mathrm{mM} \mathrm{KCl}$ of $\mathrm{pH} 4(0.5 \mathrm{mM}$ acetic acid). The fit with the theoretical Eq. (15) gives $\tau=10.25 \mathrm{~s}$. that the lysozyme concentration in the membrane must be more than $3.1 \mathrm{mM}$, resulting in a positively charged membrane. This must be caused by the adsorption of the positively charged lysozyme to the negatively charged membrane. It can be concluded that the response of this device is exclusively caused by chloride depletion at the electrolyte-membrane interface. A good fit was obtained with Eq. (15) for $\tau=10.25 \mathrm{~s}$, as is shown in Fig. 8. The experiment furthermore proves that the used combination of lysozyme concentration and incubation time is amply sufficient to detect the presence of lysozyme.

Since the proton charge of lysozyme decreases with increasing $\mathrm{pH}$, the membrane fixed-charge concentration must also decrease with increasing $\mathrm{pH}$ after the adsorption of lysozyme, expressing itself in a decrease of the transition time. This theoretical prediction is confirmed in the measurements shown in Fig. 9. In the investigated $\mathrm{pH}$ range the transition time does not decrease to the value for a bare membrane, which is also shown. This is according to expectation, since the isoelectric point of lysozyme lies around $\mathrm{pH} \mathrm{11}$, and a rest charge from the membrane itself will be present at $\mathrm{pH} 8$.

The influence of the incubation time and the lysozyme concentration on the device response

Immediately after incubation for 5, 10 and $15 \mathrm{~min}$ in solutions containing $5,0.5$ and $0.05 \mathrm{mg} / \mathrm{ml}$ lysozyme $\left(3 \times 10^{-4}-3 \times 10^{-6} \mathrm{M}\right)$, chronopotenti-

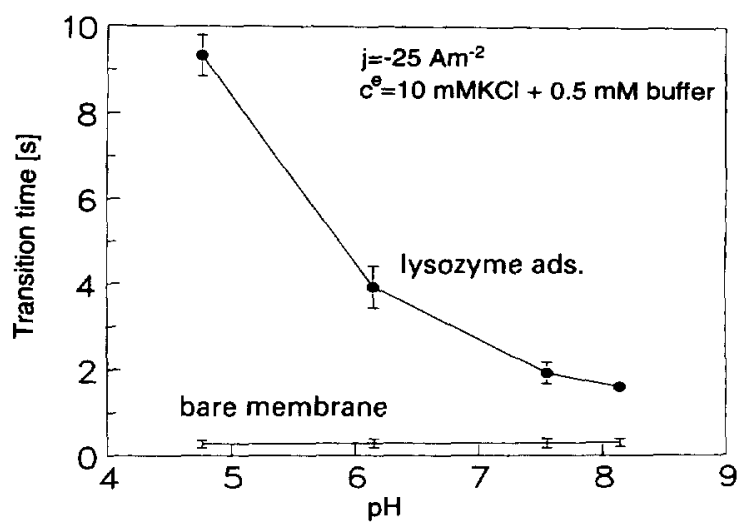

Fig. 9. The measured transition times of devices both with bare membranes and with membranes with adsorbed lysozyme measured as a function of $\mathrm{pH}$. Measurement solutions contained $10 \mathrm{mM} \mathrm{KCl}$ and $0.5 \mathrm{mM}$ buffer. Applied current density $j=-25 \mathrm{~A} / \mathrm{m}^{2}$. 


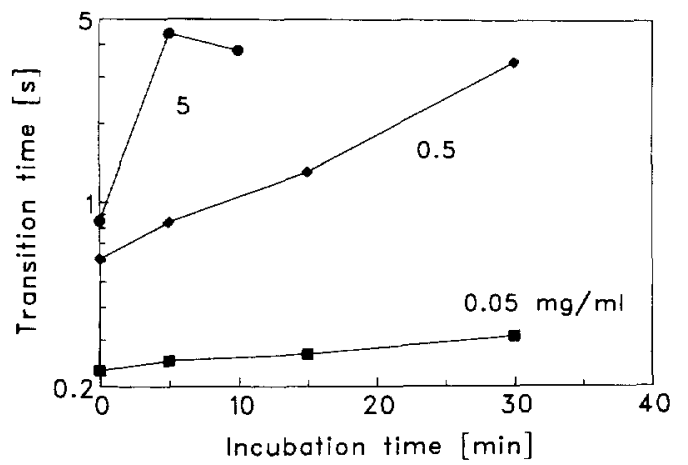

Fig. 10. The effect of the cumulative incubation time and lysozyme concentration on the transition time measured in a $10 \mathrm{mM} \mathrm{KCl}$ solution of $\mathrm{pH} 4(0.5 \mathrm{mM}$ acetic acid) at $j=-25 \mathrm{~A} / \mathrm{m}^{2}$. Note the logarithmic scale for the transition time.

ograms are made at $\mathrm{pH} 4$. The measured transition times as a function of the cumulative incubation time $(5,15$ and $30 \mathrm{~min})$ are shown in Fig. 10. The figure shows that the transition time increases with the lysozyme concentration and with the incubation time, and that the curve slope increases with increasing lysozyme concentration. Lysozyme concentrations down to $3 \times 10^{-6} \mathrm{M}$ can be detected after $5 \mathrm{~min}$ of incubation. The transition times shown were measured directly after incubation, since they decreased markedly within several minutes.

The chronopotentiograms for the adsorption of lysozyme from the $3 \times 10^{-5} \mathrm{M}$ solution are shown in detail in Fig. 11. All curves can be fitted well with Eq. (23), substituting a gradually increasing transition time $\left(\tau_{1}\right.$ to $\left.\tau_{4}\right)$.

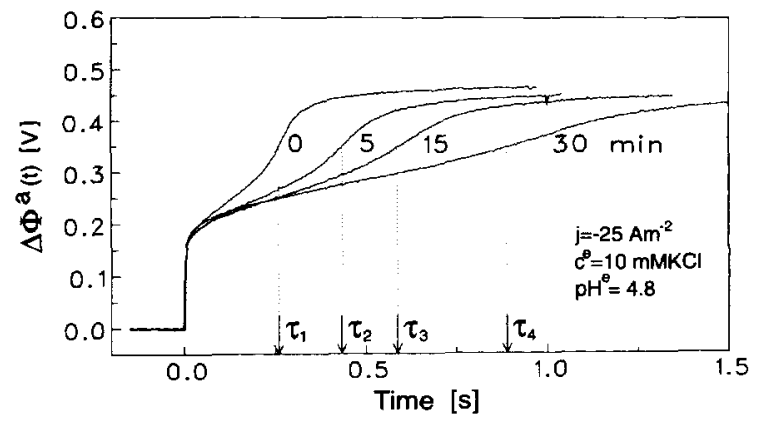

Fig. 11. The detection of lysozyme adsorption from a $3 \times 10^{-5} \mathrm{M}$ solution. $j=-25 \mathrm{~A} / \mathrm{m}^{2}$ in $10 \mathrm{mM} \mathrm{KCl}$ at $p H^{\mathrm{e}}=4.8(0.5 \mathrm{mM}$ acetic acid $)$.

\section{The influence of the current density on the transition time}

It has been shown that the transition time increases with the amount of adsorbed lysozyme. It was also shown that this increase is more steep at the current density of $-20 \mathrm{~A} / \mathrm{m}^{2}$ than at the current density of $-30 \mathrm{~A} / \mathrm{m}^{2}$. The behaviour of the membranes in this respect was investigated by incubation in a solution of $3 \times 10^{-5} \mathrm{M}$ $(0.5 \mathrm{mg} / \mathrm{ml})$ lysozyme and subsequent measuring at different current densities, looking at the increase of the transition time with the incubation time. Fig. 12 shows the transition times measured as a function of the incubation time for a typical device. Evidently the curve slope increases with decreasing current density $j$, so that a low current density is most suitable for the detection of lysozyme adsorption.

\section{The membrane-covered $\mathrm{Ag} / \mathrm{AgCl}$ electrode as a protein sensor}

Using incubation times of 5 and $30 \mathrm{~min}$ in lysozyme-containing solutions with a concentration increasing from $10^{-6}$ to $3 \times 10^{-4} \mathrm{M}$, the transition times were measured at a current density of $-25 \mathrm{~A} / \mathrm{m}^{2}$. The sensitivity for lysozyme adsorption depends strongly on the initial value of $\tau_{\mathrm{m}}$ (see Fig. 5), which in turn depends on the Donnan ratio (Eq. (20)) and thus on the membrane fixed charge concentration (Eq. (16)). Because the membranes are typically hand-made, they may differ from device to device, resulting in different values for the fixed charge concen-

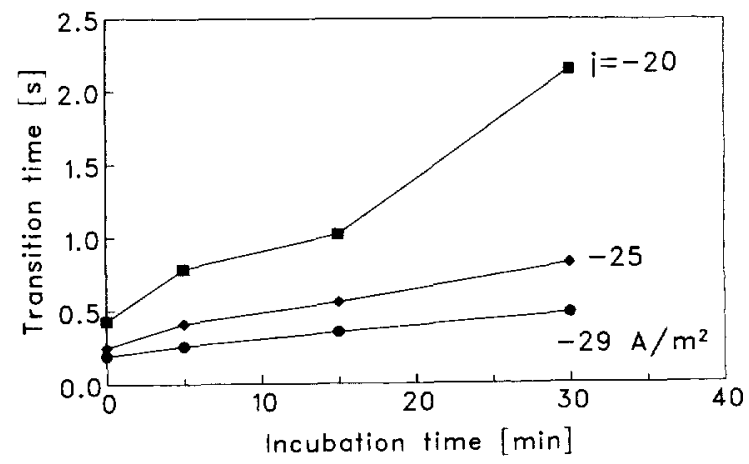

Fig. 12. The influence of the current density $j$ on the measured transition times on incubation in a lysozyme containing solution of $3 \times 10^{-5} \mathrm{M}(0.5 \mathrm{mg} / \mathrm{ml})$. Lysozyme adsorption causes the largest increase of the transition time at $j=-20 \mathrm{~A} / \mathrm{m}^{2}$. 
tration and thus for $\tau_{\mathrm{m}}$. Therefore, from 10 devices three were selected with the same initial value of $\tau_{\mathrm{m}}$ (i.e. the same set point). These three devices were each separately used for different lysozyme concentrations, $10^{-6}, 10^{-5}$ and $3 \times$ $10^{-4} \mathrm{M}$, during the indicated incubation times. Fig. 13 shows the change of the transition time, $\Delta \tau_{\mathrm{m}}$, relative to its value before incubation as a function of the lysozyme concentration. The dashed lines suggest a linear behaviour.

Since the transition times obtained are reproducible within $20 \mathrm{~ms}$, we expect the detection limit of this method to be about $5 \times 10^{-7} \mathrm{M}$ lysozyme at an incubation time of $5 \mathrm{~min}$ and less than $10^{-7} \mathrm{M}$ at the incubation time of $30 \mathrm{~min}$. For practical applications reproducible membranes should be made with an optimal value for $\tau_{\mathrm{m}}$ in relation to the range of analyte to be measured.

\section{CONCLUSIONS AND DISCUSSION}

In this paper the chronopotentiometric response of a bare $\mathrm{Ag} / \mathrm{AgCl}$ electrode was compared with that of a $\mathrm{Ag} / \mathrm{AgCl}$ electrode which was covered with a porous membrane containing fixed negative charges. The latter device exhibited a transition time of the order of $300 \mathrm{~ms}$, whilst the bare device showed a transition time of the order of $10 \mathrm{~s}$. This effect could be theoretically explained based on the Donnan effect and the calculations agreed with the experimental observations. In addition it was shown that the transition time could be modulated with the adsorption of positively charged protein molecules, with

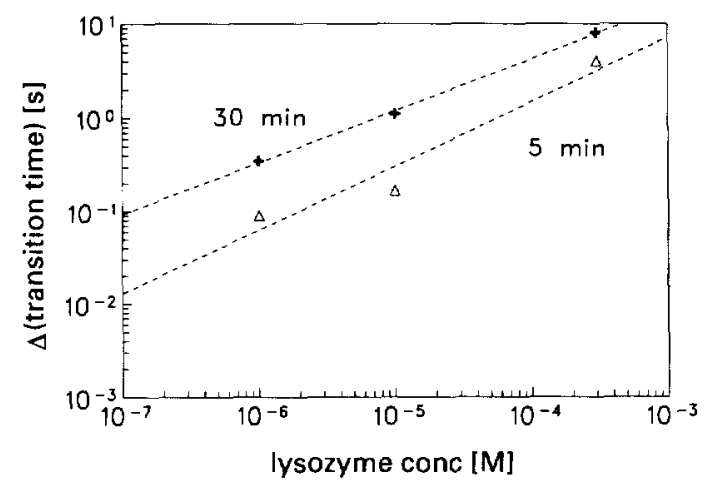

Fig. 13. Measured change in the transition time as a function of the lysozyme concentration in the incubation solution for two incubation times. Measurement solution: $c^{\mathrm{e}}=10 \mathrm{mMKCl}, \mathrm{pH}^{\mathrm{e}}=4.8(0.5 \mathrm{mM}$ acetic acid). $I=-25 \mathrm{~A} / \mathrm{m}^{2}$. lysozyme as an example. In this way protein concentrations can be measured, although limited to positively charged proteins. This is the result of the fact that the system is based on the depletion of small anions $\left(\mathrm{Cl}^{-}\right.$ions in this case) of which the initial concentration in the membrane is set by the Donnan effect, making the anion concentration minority carriers. Therefore the doping of the membrane with fixed charges can only be negative in this case. From this set point only a modulation with positively charged molecules is possible. It will be clear that also in the case of the chronopotentiometric device as described in this paper, a complementary device is possible. In that case a cation actuator should be used and the membrane fixed charges should be positively charged, and negatively charged proteins can modulate the transition time. As a cation actuator doped polypyrrole or Prussian Blue might be suitable candidates.

The chronopotentiometric experiment as described in this paper belongs to the class of stimulus/response experiments, as mentioned in the Introduction. The stimulus is an electric current, the response a voltage change from which a transition time can be determined. In this case no information can be drawn from the system in equilibrium, but the dynamic response contains the information. This is similar to other stimulus/response measurements such as the ionstep (Schasfoort et al., 1990). In the latter case a membrane-covered ISFET has to be 'stimulated' with a sudden change in salt concentration, whilst the transient change in $\mathrm{pH}$ is the response. Making the stimulus is in that case rather complicated, because it makes the use of stimulus solutions, pumps and valves necessary. It will be clear that the chronopotentiometric system as described in this paper is in this respect much more elegant, because it uses an electric current as the stimulus. Moreover, the actuator and the sensor are one and the same device, the most intensive integration one can think of. It will therefore be worthwhile investigating the value of chronopotentiometric stimulus/response measurements in more detail and under more practical circumstances than was possible in this paper.

\section{ACKNOWLEDGEMENTS}

We gratefully acknowledge the financial support of the Dutch Foundation for Fundamental Research on Matter (FOM). 


\section{REFERENCES}

Audinos, R. and Pichelin, G. (1988) Characterization of electrodialysis membranes by chronopotentiometry. Desalination 68, 251-263.

Bard, A. J. \& Faulkner, L. R. (1980). Electrochemical Methods, Fundamentals and Applications. John Wiley, New York.

Bergveld, P. (1991) A critical evaluation of direct electrical protein detection methods. Biosensors \& Bioelectronics 6, 55-72.

Donnan, F. G. (1924) The theory of membrane equilibria. Chem. Rev. 1, 73-90.

Eijkel, J. C. T. (1995). The potentiometric detection of adsorbed protein by stimulusresponse measurement techniques. PhD Thesis, University of Twente, Enschede, The Netherlands.

Eijkel, J. C. T., Olthuis, W. Kolev, S. D. \& Bergveld, P. (1997). Measuring Donnan-related phenomena using a solid-state ion sensor and a concentrationstep method. J. Membrane Sci. 127, 203-221.
Hall, E. A. H. (1990). Biosensors. Open University Press, Buckingham.

Ives, D. J. G. \& Janz, G. J. (1961). Reference Electrodes, Theory and Practice. Academic Press, New York.

De Körösy, F. (1975) An amphoteric ion-permselective membrane. Desalination 16, 85-103.

Lal, L., Thirsk, H. R. and Wynne-Jones, W. F. K. (1951) A study of the behaviour of polarized electrodes, part I.-The silver/silver halide system. Trans. Faraday Soc. 47, 70-77.

Levich, V. G. (1969). Physicochemical Hydrodynamics. Prentice Hall, London.

Schasfoort, R. B. M., Kooyman, R. P. H., Bergveld, P. and Greve, J. (1990) A new approach to ImmunoFET operation. Biosensors \& Bioelectronics 5 , 103-124.

Tanford, C. and Wagner, M. L. (1954) Hydrogen ion equilibria of lysozyme. J. Am. Chem. Assoc. 76, 3331-3336.

Turner, A. P. F., Karube, I. \& Wilson, G. S. (1987). Biosensors, Fundamentals and Applications. Oxford University Press, Oxford. 